\title{
Edgardo Pesante: \\ re-construcciones del intelectual santafesino
}

\author{
Carla Perna \\ Ivana Galetti • \\ Universidad Nacional del Litoral
}

\section{Resumen}

La presente investigación se desarrolla en el marco del proyecto CAI+D (en curso) "Tradiciones selectivas: trazo(a)s presentes y emergentes de la migración italiana y francófona de la ciudad de Santa Fe» (FHUC-UNL) y tiene por objeto un primer acercamiento a la figura del escritor santafesino Edgardo Pesante como «intelectual», atendiendo a la complejidad que dicho concepto condensa. Para ello se trabajará sobre tres aristas: sus facetas como escritor, gestor de políticas de promoción cultural (específicamente literarias) y crítico. Esta aproximación, si bien se aclara primitiva, resulta de interés para pensar no sólo los lindes o puntos de contacto con otros agentes culturales de la provincia de Santa Fe, sino también la importancia que su figura de intelectual constituye para la región.

\section{Palabras clave}

· Edgardo Pesante · intelecual · agente cultural

\footnotetext{
- Estudiante del profesorado y la licenciatura en Letras. Integrante del Centro de Estudios Comparados y del CAID «Tradiciones selectivas: trazo(a)s presentes y emergentes de la migración italiana y francófona de la ciudad de Santa Fe». Desde el 2013 se desempeña como colaboradora en el área de edición y archivo de datos en el Portal de la Memoria Gringa. Desde el 2014 al 2017 se desempeñó como Ayudante Alumna de la cátedra de Literatura Francesa e Italiana de la Facultad de Humanidades y Ciencias de UNL. - Profesora de Letras (FHUC - UNL). Cursante de la Licenciatura de Letras. Realizó adscripción en investigación en Semiótica General (año 2012) y en docencia en Literaturas Francesa e Italiana (año 2014). Desde el año 2013 integra el Centro de Estudios Comparados. Es responsable de trabajos de edición y transcripción y coordina la página web y actividad «Miradas Cruzadas. Experiencias de intercambios académicos». Becaria BAPI 2017 para el Programa de Estudios Sobre Migraciones. Integra el CAI+D «Tradiciones selectivas: trazo(a)s presentes y emergentes de la migración italiana y francófona en la ciudad de Santa Fe» bajo la dirección de Adriana Crolla.
} 


\begin{abstract}
This research is developed within the $\mathrm{CAI}+\mathrm{D}^{1}$ project (in progress) «Selective traditions: trace(s) present and emergent of Italian and french migration of the city of Santa Fe» (FHUC-UNL) and has the object of a first approach to the figure of the writer from Santa Fe Edgardo Pesante as «intellectual», taking into account the complexity that this concept condenses. Departing from this aim, three aspects will be analized: his dimensions as a writer, manager of cultural promotion policies (specifically with regional and young literature) and reviews. This approach (although it is clarified primitive), tries to focus to think not only the boundaries or points of contact with other cultural agents of the province of Santa Fe, but it also indicates the importances of Pesante through the region intellectual field.
\end{abstract}

\title{
Key words
}

- Edgardo Pesante $\cdot$ intellectual $\cdot$ cultural agent

\section{Un punto de partida}

Durante el proceso de búsqueda de producción crítica en torno a Pesante, se detectaron investigaciones que configuran aportes a una disposición primitiva del autor respecto a su condición de escritor y crítico o lo que en términos de Chávez y Cóceres se denomina «cuadro de situación» en torno al «sistema literario santafesino» (en Chávez y Cóceres, 2015:80). Tomando las apreciaciones que de aquí se desprenden, creemos fundamental identificar su rol al interior del campo intelectual santafesino para luego hacer aportes sobre la configuración de una «geoestética» (Crolla, 2015), recuperando del olvido algunas de las múltiples entradas y aportes de Pesante al campo cultural.

Nos centraremos especialmente en las apreciaciones vinculadas al recorrido investigativo del comparatismo en línea con el $\mathrm{CAI}+\mathrm{D}$ del que formamos parte, priorizando en aquellas acciones «repensar (...) la memoria y sus inscripciones discursivas en el complejo literario santafesino como un espacio de construcción presente y de la identidad local» (Crolla, Zenarruza, 2015:15-16). 


\section{Huellas del intelectual en los circuitos culturales santafesinos}

En el marco del festejo de las Bodas de Oro de ASDE (Asociación Santafesina de Escritores) El Litoral publica en sección «Nosotros» (a cincuenta años del nacimiento de la ya mencionada organización) un artículo denominado «Cumple años la casa de los escritores», en el cual se identifican ciertos conceptos sobre los que nos detendremos para pensar las re-construcciones, en particular, de Edgardo Pesante como intelectual santafesino.

Como puntapié para ese desarrollo, el artículo proporciona datos precisos que ciñen las acciones del ya mencionado autor dentro de un período que, al decir de Cañón (2003) corresponde a los ‘60: «Hace 50 años nacía en Santa Fe una organización que marcaría huellas en la historia de las letras de la ciudad. La Asociación Santafesina de Escritores surgía el 19 de octubre de 1955» (Extraído de El Litoral, año 2005).

Suma a esta localización espacio temporal los deseos y fines que identifican a los participantes de la asociación, con una claridad que permite continuar la línea de supuestos a analizar "por voluntad de un grupo de intelectuales deseosos de formar -en tiempos tan conflictivos como aquellos-, una entidad que nuclee, promueva y divulgue la literatura de la zona litoral» (Extraído de El Litoral, año 2005).

Sobre esta última parte del artículo, se recupera especialmente la construcción de una literatura específica, que selecciona en particular las propuestas de la zona santafesina y se dedica a la transmisión, centralización y divulgación de la misma hace ya más de medio siglo. Un espacio de reflexión obliga a interrogarnos acerca de qué efectos promueve un intelectual al interior de un proyecto, aunque inicialmente de forma tangencial (como fue el caso en ASDE) ${ }^{2}$, como así también las proyecciones futuras que se constituyen a partir de ese impacto, derivas y potencialidades de este y otros intelectuales con y en otros campos de acción.

Una investigación que resulta útil para contextualizar la aparición de espacios culturales a cargo del propio agente, es El cuento literario en Santa Fe, financiado por la Dirección General de Cultura, donde se hace manifiesta esa «eclosión cultural» posterior al derrocamiento de J.D. Perón del gobierno. Allí, es interesante la aclaración y el interés que se coloca en la zona literaria santafesina eclosionada al mencionar que «no está exenta Santa Fe. Muy por el contrario, se diría que la ciudad capital de la provincia vive un período de intensa actividad» (Pesante, 1969).

En el marco de esa intensidad declarada, identificando un momento histórico de porvenir cultural, clarifica las numerosas creaciones de asociaciones donde menciona la Asociación Santafesina de Escritores advirtiendo «que encaran todo tipo de actividades de orden cultural y artístico, necesarias muchas, aunque, como es natural, el proceso de inflación no deja de aparecer» (Ibíd.).

Interesa advertir algunas marcas federalistas con las que se declara el espíritu de la investigación, en tanto podrían ser un punto de anclaje a la hora de pensar en los intelectuales y las producciones de los mismos en ese contexto de acción, ya que al «delinear su centro sin tocar la dominante cultural del momento, sin cruzarse con ella, no implica negar la productividad que estos grupos generan en la historia literaria nacional» (Cañón, 2003: 110). La doctrina política es un punto sobre el cual se parte ya que instala condicionantes en torno al juego del centro por sobre la periferia y asimismo es indispensable para determinar (independientemente de las pujas geopolíticas) la productividad del Litoral argentino como espacio formador de huellas literarias. 


\section{De legados y palabras}

Es entonces la Asociación Santafesina de Escritores (ASDE) uno de los focos de encuentros y prácticas escriturales entre intelectuales y germinador de autores consagrados, como Enrique Butti, Nora Didier, Miguel Ángel Gavilán, entre otros.

Al decir de Nora, «él era el maestro, el que nos guiaba (...) uno de sus objetivos era darle lugar a todos los jóvenes que quisieran sumarse al hecho literario» (Didier, 2017) ${ }^{3}$.

Dentro de ASDE, Pesante

se manifiesta a través de un accionar permanente en favor de las letras, mediante certámenes literarios conocidos en toda la región, ciclos de conferencias, talleres, antologías orales y escritas, y la participación de la entidad en las Ferias del Libro de Santa Fe y de Buenos Aires. (Extraído de El Litoral, 2005)

Las acciones tangenciales que complementan el proceso del Pesante Lector y productor de Literatura (haciendo referencia concreta a su labor en periodismo, docencia ${ }^{4}$, edición ${ }^{5}$ y gestión cultural durante sus períodos activos en la Asociación previamente mencionada) lo erigen como un caso complejo que debe extender su dominio a la noción de campo intelectual, entendido como ese «campo magnético» que «constituye un sistema de líneas de fuerza: esto es, los agentes o sistemas de agentes que forman parte de él pueden describirse como fuerzas que, al surgir, se oponen y se agregan, confiriéndole su estructura específica en un momento dado del tiempo" (Bourdieu, 2002:9).

Interesan los legados de este agente al campo, desde esta arista, inscriptos en una propuesta de lo que actualmente identificamos como mediación lectora y prácticas de escritura. Estos agentes, como es el caso de Pesante o Bonocuore, no se reconocen como tales sino que operan al interior de un sistema de fuerzas, que en este caso se intensifican incluso con legados y autores. La propuesta del taller construye vínculos entre personalidades de la ciudad y sus alrededores, pero además, deja bienes culturales que actualmente se conservan en la sede de la Asociación ${ }^{6}$.

En la edición del día jueves 15 de marzo de 2001, a efectos de conmemorar el vigésimo noveno aniversario de la creación del taller, el Profesor Miguel Angel Zanelli hace mención explícita del ingreso de Edgardo Pesante a la propuesta, «en agosto de 1972», quien (aclara) «significó un gran aporte a la formación del taller» (2001).

Si bien esta nota cultural opera como invitación a la participación de la comunidad (no estrictamente académica o letrada), interesa hacer una especial detención en aquellas pistas del contexto de producción del intelectual en cuestión, y los efectos que su paso por determinadas instituciones convocaron (como así también, sus bienes culturales cedidos a asociaciones). Es de ese modo, la producción de Edgardo Pesante, concebida como parte de la maquinaria que funciona como «la palabra de aliento a la palabra» (Zanelli en El Litoral, 2001).

Otro ítem central en relación con ASDE radica en el rol del agente como secretario de redacción de «La Gaceta Literaria», publicación que se concreta en el año 1981 bajo la Dirección de Luis Di Filippo y «de secretario general» se designa a "Jorge Alberto Hernández (Beto Hernández) y de redacción a Edgardo Pesante» (Didier, 2017).

En este sentido, uno de los legados que entrega a la Profesora Nora Didier es precisamente ese cargo de Secretaria de redacción, que mantiene por años. Posteriormente, esta publicación de frecuencia mensual modifica su nombre a Revista Literaria (en el año 2009) y en ella se evidencian los cambios en los directivos y secretarios respectivos. 
Actualmente, pueden consultarse los materiales accediendo a la Sede de la Asociación Santafesina de Escritores, pero además se observan influencias en otros espacios de circulación (como el reservorio de Catálogos colectivos de la Universidad de Buenos Aires).

Sea como fuere esta mutabilidad que ha experimentado el modo de nombrar el medio, la propuesta iniciada por el agente cultural aún no ha cesado de germinar, promoviendo en dicha materialidad una suerte de espacios para la presentación de voces vinculadas con la palabra.

\section{La huella Pesante en EI Litoral}

A raíz del rastreo del material disponible en la Hemeroteca $D i$ gital Fray Francisco de Paula Castañeda (archivo de la provincia de Santa Fe), realizada como una primera instancia para el proyecto CAID ya mencionado, podemos establecer que entre los años 1960 y 1979 Edgardo Pesante no sólo publicó cuentos y relatos, veintidós en total, sino que además le dedicó espacio a la crítica literaria, publicando veintiún artículos referidos tanto a escritores de la «zona» (Mateo Booz, Carlos Eduardo Carranza, Lamothe) como a escritores nacionales (J.L. Borges) e internacionales (Buzzati, Hudson), refiriendo en ellos a temas de la literatura en general y del cuento en particular. Algunos de los cuentos publicados en este periódico fueron reunidos en el libro El día que no amaneció editado por la editorial Colmegna en 1975. Allí aparecen recopilados "El gran archivista no tardará...»" «El espejo», «Las inocentes palomas» y «El juicio final» junto a otros relatos que habían sido publicados en La prensa, revista Ser de Concepción del Uruguay y La voz del interior de Córdoba.

De lo publicado por estos años, en este trabajo nos vamos a centrar en tres artículos y un cuento que reflejan una lectura de lo «local» acompañada de la acción de rescate de ciertos escritores de la «zona» que intenta poner en valor, en este caso, la figura de Carlos Eduardo Carranza ${ }^{8}$, a quien dedica dos publicaciones a lo largo de su trabajo en el diario: «Un escritor olvidado» (1963) y «Para una revaloración de Carranza» (1975). El otro artículo, parte del corpus trabajado aquí es «Valoración del cuento" (1964) donde trata de definir este género en contraposición al relato y a la novela. Y, por último, «iAdiós Colastiné!» publicado en diciembre de 1963.

\section{Lecturas de «lo local»}

La pregunta que abrimos aquí es de qué manera la figura de Edgardo Pesante contribuye a la configuración de una geo-estética en la región imaginaria de la Pampa Gringa. Para tratar de esclarecer o arrojar una respuesta tentativa indagaremos primero en una lectura que de nuestra localidad hace Pesante a través de una impronta crítico-literaria que intenta salvar del olvido a escritores de la «zona»" que se leen por fuera del canon ${ }^{10}$ santafesino, revalorizando además el género cuento - mal considerado para su gusto como «el hermano menor de la novela» por aquella época. 
Tanto en «Un escritor olvidado» como en "Para una revaloración de Carranza», Pensante liga la figura de Carlos Eduardo Carranza a la de Mateo Booz ${ }^{11}$ poniendo de manifiesto, en primer lugar, su amistad y sus elecciones tanto literarias (en cuanto al regionalismo que ambos escritores practicaron), como profesionales (los dos fueron periodistas), pero salvando una diferencia radical: uno se afirma, con su vasta obra, en el canon literario de la época, y quien fuera el autor de Abalorios $^{12}$ cae estrepitosamente en el olvido. El «tono irónico pero no hiriente» de los cuentos que se reúnen en Abalorios son otra muestra de las características que unen a Carranza y Booz. Por otro lado, recuerda la sociedad literaria que ambos integran hasta 1923. En colaboración publican en folletín, en el diario La Nación de Buenos Aires, la novela El salvador de la estirpe y estrenan, en Santa Fe, la obra teatral Don Osorio.

Abalorios, según este autor, reúne cuentos que «intentan y logran en buena medida dar una visión de mundo provinciano — santafesino- que Carranza vivió.» (El litoral, 1975). "Faltaba una prueba» es uno de los veinte cuentos reunidos en Abalorios y en el cual Pesante se detiene por ser «un trabajo comprometido de Carranza», entre otros que tal vez resultan «anecdóticos» o poco serios y de los cuales un "crítico exigente podría considerar excesivamente 'provincianos'» en un sentido peyorativo. "Faltaba una prueba», en cambio, es "la historia de la crisis moral de un periodista», acción que transcurre en "una Santa Fe un poco nebulosa» ${ }^{13} \mathrm{y}$ en el último año de la primera guerra mundial (1918). A partir de este pequeño análisis o comentario, por un lado, Pesante abre camino e intenta poner en valor ciertos elementos de Abalorios para su reedición y un posterior estudio que ponga de manifiesto su característica de patrimonio cultural; y, por otro lado, «sólo quiere llamar la atención sobre un escritor que, si bien no realizó aportes notables a la literatura, no se merece el olvido a que parece condenado».

Para Pesante, el cuento literario santafesino comienza allí, con Carranza, Mateo Booz y Alcides Greca ${ }^{14}$, así lo expone en «Para una revaloración de Carranza», considerándolos precursores de las generaciones posteriores:

Carranza, con Alcides Greca («Cuentos del comité», 1931) y Mateo Booz («Santa Fe, mi país», 1934) son los fundadores del cuento literario en Santa Fe. Se inscriben en la corriente regionalista que nace del tradicionalismo de Joaquín V. Gonzalez, se afianza en el costumbrismo crítico de Roberto J. Payró y alcanza su cumbre continental con Horacio Quiroga. Los cuentistas santafesinos anteriores a 1940 recogen esa herencia y le ponen un sello provincial digno. (Pesante, El litoral, 1975)

...una figura como la de Mateo Booz, esencialmente cuentista, que desde Santa Fe trasciende al plano nacional, gravita sobre su generación y las posteriores, no sólo en cuanto a seguidores en estilo y enfoque - lo que posiblemente sea relativo—, sino, sobre todo, en lo que se refiere al cultivo del género en sí, a la preferencia por el cuento como medio de expresión de los escritores santafesinos. (Pesante, El cuento literario en Santa Fe, 1969: 8)

Pesante define - y defiende - el sistema literario del cuento moderno ${ }^{15}$ santafesino proponiendo como origen Santa Fe, mi pais (1934) de Mateo Booz y Abalorios (1935) de Carlos Eduardo Carranza, quienes reciben influencia tanto de Maupassant, Chejov, Kipling y Poe, como de Payró y Quiroga. Sitúa, además, en la figura Domingo G. Silva el antecedente más lejano del cuento santafesino. 
El trabajo que Pesante comienza con los artículos publicados en el diario El Litoral son consecuentes con su propuesta y concluyen en una "monografía» titulada El Cuento Literario en Santa Fe publicada en 1969 por la Dirección General de Cultura de la Provincia, en la cual arriesga, más allá del detallado sistema literario que comprende desde los ańos ' 30 hasta el "Grupo Adverbio", Trabajos I y los cuentos reunidos en 13-19 en 1967, que «el cuento sería el género literario que mejor representa a Santa Fe» (Pesante, 1969:8).

Acaso haya sido - y quizá lo sea todavía - el género literario más apto para reflejar al hombre y a la circunstancia de Santa Fe. Nuestro pasado, la historia lugareńa, es de una riqueza poco común, por su variedad e importancia, como para que la novela haya podido abarcarlo. Es sabido que la diferencia entre cuento y novela no es cuestión de cantidad de páginas, sino que, contrariamente al cuento, la novela ofrece visiones panorámicas, totalizadoras, siendo que el cuento, en cambio, toma fragmentos, valorizándolos con la intensidad que exige su técnica en el tratamiento de los temas. (Ibíd.)

Éste es otro de los temas tratados en sus publicaciones ${ }^{16}$. Para poder exponer tal sistema literario, debe tomar una posición. La empresa que lleva adelante Pesante tiene que ver con su rol de agente cultural a través de una acción de rescate y puesta en valor. Elige, en primera instancia, el género del cual se va a ocupar tanto en sus trabajos de investigación como de vocación poética: el cuento por sobre la novela; $y$, en segunda instancia, aquellos narradores santafesinos que fuera del canon eligen también el cuento que para Pesante «refleja» el hombre y su circunstancia lugareña. El mismo Pesante, fuera de canon, también narra Santa Fe.

En «iAdiós, Colastiné!» la ciudad es construida como un gran espacio que cruzando la laguna distancia a los amigos, en contraposición a Colastiné, donde hay encuentro y cercanía: «La ciudad lo atemorizaba, porque disgregaría a sus vecinos, modificando el paisaje cotidiano, vivo, hecho de encuentros, de saludos, de charlas y de juegos» (Pesante, El Litoral, 1963: 8). La inundación expulsa al Rubio y su familia desde las orillas a Santa Fe, los presiona al progreso. El ferrocarril se construye como metáfora de ese camino, que pasa por ese espacio de todos y de ninguno, que no acepta todavía alambrado ni mojones, que une y sigue siendo encuentro: el río, el Paraná; ese camino que atrás deja la niñez, la costa y el peligro por una ciudad con nuevo puerto. Al Rubio, lector y entusiasta de las historias gringas y criollas que le narraban los viejos sobre Garibaldi, los piratas del Mediterráneo y los naufragios, le atemorizaba la ciudad porque las aventuras prefiere vivirlas en la imaginación. Al llegar, lo antes percibido como distante entre faroles oscilantes recibe al Rubio y su familia en la muchedumbre y el abrazo de los conocidos. Las luces, la prisa del tren, que desde la costa salía a paso de hombre, las explosiones, las estrellas fugaces y las risas traen como metáfora del progreso el año nuevo de 1912. Colastiné pasa a ser un recuerdo, el Rubio quiere y sueña conocer el nuevo puerto de la ciudad «abierto a la esperanza».

Su impronta crítico literaria es una manera también de posicionarse como narrador dentro de su propio sistema. En El Cuento Literario en Santa Fe, luego de citar los distintos autores de las diferentes épocas que para él forman parte de este sistema, en primer lugar, retoma el trabajo 13-19, donde es incluido como uno de los 13 autores que escriben las 19 narraciones que allí se publican. Y, por otro 
lado, cita como uno de los más importantes medios de circulación de la época el periódico y la editorial de El Litoral, espacio en el cual publica tanto artículos de crítica como cuentos y relatos. Prepara, a través de su trabajo crítico, los cimientos que sostienen su trabajo de escritor alzando las banderas sobre un espacio de vacancia que pone en discusión.

El proyecto crítico de Pesante es una acción de disputa por el reconocimiento y la legitimidad dentro del campo literario. Pesante lucha desde fuera del canon por este capital simbólico a través de los medios de circulación e instituciones que pertenecen al campo de producción cultural: revistas, editoriales y diarios.

... cada campo social se puede describir como un sistema de posiciones, cada una de las cuales se define por sus relaciones con otras posiciones. Pensar en términos de campos, ha insistido Bourdieu, implica adoptar una forma de pensamiento relacional (...) que abandone la noción de «propiedad sustantiva» por la de "propiedad relacional». Un campo social es también un «sistema de relaciones de fuerza» y un «espacio de luchas». (Pastormerlo, s/f: 96)

El sistema que conforma constituye uniformidad en la red de relaciones de un universo diverso y heterogéneo. Este sistema de lectura progresiva y lineal del cuento moderno santafesino permite a Pesante posicionarse en este «espacio de luchas». Construir tal sistema, supone remontarse a un «origen», el del cuento moderno santafesino, con Mateo Booz y Carlos E. Carranza, proveyendo de un sistema de relaciones que se manifieste a través de los préstamos e influencias (Poe, Payró, Quiroga, etc.), y que circule por las instituciones ya pertenecientes al campo literario y cultural que lo abarca. Siguiendo a Pastormerlo, los escritores más allá de pertenecer a un determinado contexto social, habitan también «y ante todo un mundo social más reducido y específico integrado por otros escritores, críticos, editores e instituciones propias de esa zona social diferenciada y ya provista para entonces de un importante grado de autonomía» (Pastormerlo, s/f: 98).

En conclusión, a raíz del análisis de una selección del material archivado por la provincia en la hemeroteca digital de las publicaciones que haya hecho Edgardo Pesante para el diario El Litoral, uno de los más importantes medios de circulación de la época, vemos un especial interés del autor sobre el género cuento, recuperando aportes teóricos y críticos sobre el mismo, como así también una lectura y escritura intertextual que recupera influencias tanto locales como extranjeras, en particular italianas, a través de Buzzati y Pirandello ${ }^{17}$. Pesante lleva adelante una lectura de nuestra localidad a través de una impronta crítico-literaria que intentando salvar del olvido a escritores de la "zona» que se leen por fuera del canon, poniendo en valor además el género cuento.

\section{Punteos finales}

Si bien hemos reconocido varias aristas del caso Pesante, creemos que este proceso de re-lectura aún no está terminado. Resulta clave reconocer esta peculiar condición de agente cultural, ya que permite indagar en aquellos contactos que dicho intelectual — poco estudiado ${ }^{18}$ - estableció con asociaciones y espacios de producción de contenidos culturales. Si bien 
los archivos, textos editados, borradores y guiones radiales son útiles a la hora de actualizar el caso, las producciones e investigaciones mínimas disponibles durante el contexto de producción del agente resultaron de suma utilidad para la etapa de selección, como así también aquellas entrevistas con otros agentes cercanos que ofician al punto de "traductores» de la experiencia, de la maquinaria del recuerdo y los retazos de memoria. Todo ello opera aquí como antecedente para recopilar en detalle el accionar del intelectual escogido.

La praxis a la que convoca el Pesante docente y escritor como parte de un sistema literario de la periferia es posible imaginarla del mismo modo que su personaje en Gauguín, como un sujeto que «escribía de mañana, de tarde, de noche» (Pesante, s/f:9). Porque si algo es seguro son sus intereses sociales, apostando por una política de los bordes, valorando y enalteciendo a los escritores silenciados, a los jóvenes, a los periféricos. Comulgando junto a la comisión de la Asociación Santafesina de Escritores con un «espíritu de compromiso con los valores éticos y la gallardía.» (Extraído de El Litoral, 2005).

Es posible imaginar, también, esa praxis materializada con la palabra justa, diseccionando las propuestas de los talleristas, otorgando «lugar a todos los jóvenes que quisieran sumarse al hecho literario» (Didier, 2017), vislumbrando las potencialidades de borradores que se han transformado en textos editados y publicado.

Esta instancia permite reconfirmar en Pesante el sentido de intelectual que configura Said, no ya un pacificador, no ya un fabricante de consenso, sino alguien que rechaza fórmulas sencillas, estereotipos, y lugares comunes. Es ese torrente de esfuerzos, de energías «racionales» y luchas lo que dota a Edgardo Pesante de vocaciones intelectuales de forma total (Said, 1996). 


\section{Notas}

${ }^{1}$ Course of Action for Research and Development of UNL

${ }^{2} \mathrm{Si}$ bien Edgardo Pesante no integra el acta fundacional de la Asociación en cuestión, materializada en la Biblioteca Cosmopolita, es sucesor de Leoncio Gianello en la presidencia. Datos como este fueron recolectados en una entrevista realizada a la ex presidenta de la ASDE, la Profesora y escritora Nora Didier.

${ }^{3} \mathrm{La}$ reconstrucción de su activismo cultural al interior de dicha institución se lleva a cabo en gran parte gracias a la entrevista realizada a Nora Didier, como así también a aquellas menciones en el periódico donde el mismo Edgardo Pesante llevó a cabo sus manifestaciones sobre los acercamientos de jóvenes a las propuestas de escritura que junto a Zanelli encarnaban en los encuentros del taller literario. La entrevista se realizó en el ańo 2017 a la escritora en el marco del proyecto de investigación, a fines de justificar la potencialidad del intelectual no únicamente en ámbitos universitarios e instituciones legitimadas a nivel nacional, sino también aquellas Asociaciones del interior.

${ }^{4}$ Se hace referencia a los ingresos de Edgardo Pesante en la docencia en el artículo de El Litoral, al mencionar la creación del Taller Literario de ASDE en 1972 y, se reconoce que «posiblemente sea éste el más antiguo del interior del país» (2005). Si bien el taller comienza con la designación de Miguel A. Zanelli por parte de la comisión directiva para llevar adelante el trabajo de mediación y producción de textos literarios, cinco meses después se reconoce la incorporación de Pesante en este espacio. El epígrafe hace mención a las experiencias de trabajo con esta figura por parte de la Prof. Nora Didier, en una entrevista realizada por las autoras de este trabajo durante el año 2017.

${ }^{5}$ Identificamos la potencialidad de La Gaceta Literaria como foco productor y transmisor (al menos, al interior de la zona santafesina) y el valor de Edgardo Pesante como integrante de la misma en sucesivas gestiones de comisión.

${ }^{6}$ En la publicación conmemorativa de Diario El Litoral (año 2005) se hace mención al legado de Luis Di Filippo, «cuyo material —disponible para socios y la comunidad en general - procede de las bibliotecas privadas de Julio Caminos, Edgardo Pesante y del propio Di Filippo» (2005). ${ }^{7}$ El paratexto título es modificado por «El gran archivista no tardará en llegar».

${ }^{8}$ Carlos Eduardo Carranza fue un periodista y escritor nacido en Casilda en abril de 1881. Llega a Santa Fe en 1912 a trabajar en el diario $L a$ Democracia.

${ }^{9}$ Entendemos el término «zona» desde los aportes de Crolla, quien recupera la noción saeriana "para indagar en nuestra territorialidad [...] pues nos habilita a un ejercicio crítico, estético y comparado sobre las fronteras lábiles y las condiciones geopolíticas que posibilitaron la emergencia de una cultura local en relación con matrices culturales aportadas por la inmigración italiana.» (2015:16)

${ }^{10}$ Entendemos «canon» en el sentido que lo explica Malena Botto, como «una selección que no implica meramente una lista de textos 
canónicos como resultado, sino un proceso por el cual otros escritos son apartados, si no se ajustan a los parámetros de selección, y este aspecto es crucial para la conservación y posterior "visibilidad" de los textos en una cultura» (s/f: 128)

${ }^{11}$ Mateo Booz, seudónimo de Miguel Ángel Correa, nace en Rosario en 1881 y se radica en Santa Fe en el año 1911. Se desempeñó en varios cargos públicos y ejerció el periodismo hasta el año 1920, año en que pasó a dedicarse de lleno a la escritura narrativa. Además de Santa Fe, mi pais (1934), entre sus principales obras se encuentran: La mariposa quemada, Tres lagunas, Aleluyas del Brigadier, Soldados y almaceneros, Aquella noche de Corpus, El tropel, La ciudad cambió de voz, y Gente del litoral. La película argentina Los inundados (1962), de Fernando Birri, está basada en el cuento homónimo de este escritor. Muere el 16 de mayo de 1943. ${ }^{12}$ Único libro que alcanza a publicar Carlos Eduardo Carranza el mismo año de su muerte, 1935.

${ }^{13}$ Pesante toma como hecho real de referencia de la construcción espacio-temporal el año 1915, año que en Santa Fe nevó, y de la cual el cuento se hace eco.

${ }^{14}$ Alcides Greca nace en la localidad de San Javier, provincia de Santa $\mathrm{Fe}$, en 1889. Fue escritor, abogado, periodista, jurista, profesor universitario y cineasta. Funda y dirige los periódicos El Mocoví (1909) y La pura verdad (1911) en la localidad de la que es oriundo, El Paladín del Norte (1913) y La palabra, antecedente del todavía hoy editado diario El Litoral, en la ciudad de Santa Fe. En 1909 publica su primer libro, Palabras de Pelea. En 1917 filma El último malón. Una de sus obras más importantes es La pampa gringa, novela que publica en 1936. Muere en Rosario el 16 de abril de 1956.

${ }^{15}$ Edgardo Pesante entiende el cuento moderno desde E. A. Poe, como una narración breve y de acción conclusiva.

${ }^{16}$ En «Valoración del cuento», Pesante define y rescata el género cuento a partir de las nociones de Pagés Larraya, Sakai Kazuya, Mastrángelo Carlos y Maugham William Somerset. Evoca a sí mismo a Horacio Quiroga, E.A. Poe, Guy de Maupassant, Anton Chejov, Dino Buzzati y Ray Bradbury. ${ }^{17} \mathrm{El}$ artículo publicado sobre Buzzati, «Buzzati y la juventud» (1968), se centra sobre uno de los capítulos ("Patrocinador de jóvenes») de En aquel preciso momento de este autor y la dicotomía viejo/joven que se establece en el relato a través del humor. En lo que respecta a Pirandello, en junio de 1972 Pesante publica, en el diario El Litoral, «Elegía», relato inspirado en las "Elegías renanas» de Luigi Pirandello, influencia declarada ya desde el paratexto epígrafe citando un fragmento de la misma. La cita, forma del trabajo intertextual "pone al texto en relación manifiesta o secreta con otros textos», en términos de Genette, generando a su vez una reescritura como trabajo de reinterpretación.

${ }^{18}$ Que E. Pesante quede por fuera del canon oficial o tradicional, incluso del crítico, es también un motor que debería impulsar cuestionamientos en torno a la mirada del crítico literario o del mero investigador acerca de las prácticas naturalizadas de lectura. 


\section{Referencias bibliográficas}

Altamirano, C. y Sarlo, B. (2002). Intelectuales. En Términos de la sociología de la cultura (148 a 149). Buenos Aires: Paidós.

Botтo, M. (s/f). Canon. En Amicola, J. y De Diego J. L. (dir.) Conceptos críticos de la teoría literaria del siglo XX (126 a 140). Colección «Textos Básicos».

Bourdieu, P. (2002). Campo de poder, campo intelectual. Buenos Aires: Montressor, Colección Jungla Simbólica.

Crolla, A. (2015). Territorios de la italianidad como fatalidad: una mirada desde la «zona». En Crolla, A. (ed.) Italia y Francia en Santa Fe: diversidades, legados y reconfiguraciones (15 a 25). Santa Fe: Ediciones UNL. Diario EL LITORAL (22 de octubre de 2005). Cumple ańos la casa de los escritores. Recuperado online http://www.ellitoral.com/index.php/ diarios/2005/10/22/nosotros/NOS-10.html Genette, G. (1989). Palimpsestos. Madrid: Taurus.

Gramsci, A. (1967). La formación de los intelectuales. En La formación de los intelectuales. México: Grijalbo.

Hemeroteca Digital Fray Francisco de PaUla Castañeda www. santafe.gov.ar/hemerotecadigital/articulo/portada/

Pastormerlo, S. (s/f). Campo literario. En Amicola, J. y De Diego J.L. (dir.) Conceptos críticos de la teoría literaria del siglo XX (96 a 111). Colección Textos Básicos.

Pesante, E. (1969). El Cuento Literario en Santa Fe. Santa Fe: Dirección General de Cultura de la Provincia. (s/f). Gauguin. En Concierto para la mano izquierda. Santa Fe: Ed. Colmegna.

SAID, E. (1996) Representaciones del intelectual. Buenos Aires: Ed. Paidós. 